\title{
GAIA Non-Reassuring Fetal Status Level of Diagnostic Certainty
}

National Cancer Institute

\section{Source}

National Cancer Institute. GAIA Non-Reassuring Fetal Status Level of Diagnostic

Certainty. NCI Thesaurus. Code C126847.

A classification of maternal and fetal outcomes relating to non-reassuring fetal status, developed by the Global Alignment of Immunization safety Assessment in pregnancy, based on the extent to which the diagnosis has been confirmed. 